\title{
Ab Initio Search for Hydrogen Storage Nanostructured Materials
}

Yong-Hyun Kim,* Yiyang Sun,** J. Kang*** W. I. Choi,***, Kyuho Lee, ${ }^{* * *}$ Yufeng Zhao,*** and S. B. Zhang, **

* Graduate School of Nanoscience and Technology (WCU), Korea Advanced Institute of Science and Technology, Daejeon 305-701, Korea

** Department of Physics, Applied Physics and Astronomy, Rensselaer Polytechnic Institute, Troy, NY 12180, USA

*** National Renewable Energy Laboratory, Golden, Colorado 80401, USA

Physisorption-based $\mathrm{H}_{2}$ storage is as fast and reversible as traditional high-pressure gas-tank storage because it is based on a surface reaction. By maximizing the surface area of nanoporous materials, one can store hydrogen up to $7.5 \mathrm{wt} \%$, but currently only at cryogenic temperature of $77 \mathrm{~K}$. The low operating temperature is attributed to the weak dihydrogen-material interactions. At room temperature, typical high surface area hydrogen storage nanoporous materials can store only less than $1 \mathrm{wt} \%$ of $\mathrm{H}_{2}$.

To overcome the temperature gap, one needs to design a material that has many dihydrogen physisorption sites with energy gains of $0.2-0.6 \mathrm{eV}$ per $\mathrm{H}_{2}$. Based on the first-principles computational materials science, we have proposed a series of methods to design enhanced hydrogen physisorption sites on metal-dispersed nanostructured materials, which could have energy gains of $>0.2 \mathrm{eV}$ per $\mathrm{H}_{2}$ [1-6]. At first, we could be able to propose a system that can store $\mathrm{H}_{2}$ up to 9 wt $\%$ based on theoretical consideration [1]. However, the system was too reactive to be isolated in experiment. In another effort, we have recently studied dihydrogen interaction with nanostructured materials that are synthesized in experiment already, such as metal-organic frameworks [3] and porphyrins [6]. Such materials, however, can store very minimal amount of hydrogen, namely one $\mathrm{H}_{2}$ per metal site. Therefore, in order to design optimal hydrogen storage nanostructured materials, we have to carefully balance between the reactivity and the hydrogen storage capacity of the nanostructured materials, as conceptualized.

In this talk, I will summarize our efforts to design physisorption-based hydrogen storage nanostructured materials and discuss future directions to optimize and balance dihydrogen-metal interactions and storage capacity.

This work was supported by the WCU (World Class University) program through the National Research Foundation of Korea funded by the Ministry of Education, Science and Technology (Grant No. R31-2008-000-10071-0).

References

[1] Y. Zhao, Y.-H. Kim, A. C. Dillon, M. J. Heben, and S. B. Zhang, Phys. Rev. Lett. 94, 155504 (2005).

[2] Y.-H. Kim, Y. Zhao, A. Williamson, M. J. Heben, and S. B. Zhang, Phys. Rev. Lett. 96, 016102 (2006).

[3] Y. Y. Sun, Y.-H. Kim, and S. B. Zhang, J. Am. Chem. Soc. 129, 12606-12607 (2007). 
[4] Y.-H. Kim, Y. Y. Sun, and S. B. Zhang, Phys. Rev. B 79, 115424 (2009).

[5] Y. Y. Sun, K. Lee, Y.-H. Kim, and S. B. Zhang, Appl. Phys. Lett. 95, 033109 (2009).

[6] Y.-H. Kim, Y. Y. Sun, W. I. Choi, J. Kang, and S. B. Zhang, Phys. Chem. Chem. Phys. 11, 11400 (2009).
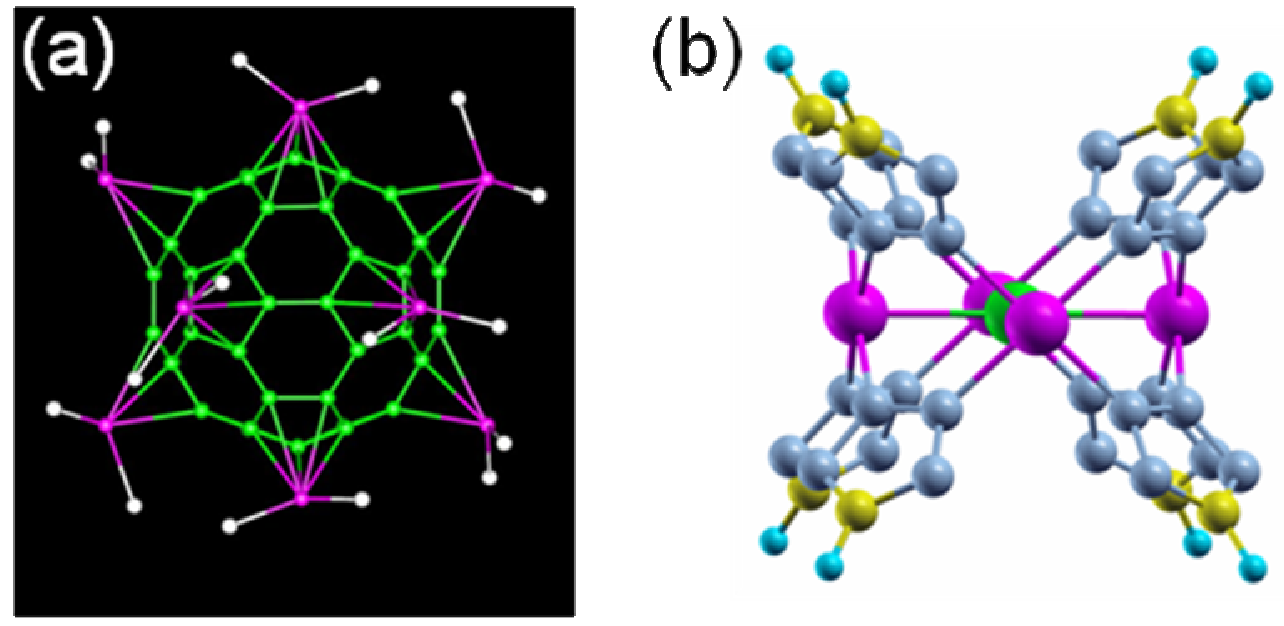

FIG. 1. Examples of hydrogen storage nanostructured materials. (a) High-capacity, but too-reactive, hydrogen storage materials, namely, transition-metal-decorated $\mathrm{C}_{60}$, which was theoretically proposed for high-capacity hydrogen storage. (b) Transition-metal-exposed metal-organic frameworks, which was experimentally synthesized and tested, but only can hold one $\mathrm{H}_{2}$ per transition metal site. 